\title{
EDITORIAL
}

\section{Claves para la postulación y escritura de artículos en revistas indexadas}

\author{
Jorge Mario Ortega Iglesias ${ }^{1}$
}

1. Editor. Universidad del Magdalena, Santa Marta, Colombia. Correo electrónico: jortegai@unimagdalena.edu.co

\footnotetext{
Q
} uienes inician estudios de posgrado tanto a nivel de maestría como de doctorado se enfrentan necesariamente con el compromiso de la divulgación y la publicación de su actividad investigativa, la cual se encuentra articulada con su proceso de formación, incluso, en algunos casos, constituyéndose como un requisito de estricto cumplimiento para obtener su grado. La divulgación y la publicación de la actividad científica, para comunidades selectas de investigadores y académicos de alto nivel, suele ser una actividad común y cotidiana, siendo parte indispensable de sus agendas y compromisos laborales. No obstante, para quienes tienen poca o nula experiencia en esta práctica investigativa, suele constituirse, por lo general, como un obstáculo y quizás un requisito de complejo logro.

¿Cómo escribir un artículo científico? ¿Qué se debe tener en cuenta para garantizar la calidad de nuestros manuscritos? ¿Cuáles son los puntos clave de todo este proceso? Podrían ser algunas de las preguntas que se hace de manera inminente el nobel "investigador", estudiante que inicia su vida en este tipo de periplos. Así pues, bajo el espíritu pedagógico que se requiere para formar en estos quehaceres, se presentan en esta editorial una serie de recomendaciones y pasos para la postulación y la escritura de artículos en revistas indexadas. Sin embargo, es preciso dejar claro que la naturaleza de estas claves no constituye una verdad sentada o una garantía para la publicación definitiva de un artículo elaborado, pues, por el contrario, hacen parte de un conjunto de recomendaciones surgidas a partir de la experiencia investigativa y las voces tácitas que muchas veces acompañan este complejo $y$, en algunos casos, largo proceso. De esa manera, este documento es una apuesta formativa en proceso de construcción continua, flexible y dinámica, para que estudiantes de pregrado, maestría, doctorado y demás académicos que inician su proceso en la publicación de artículos tengan una pauta a la hora de postular y escribir sus producciones académicas.

A continuación, se presentan algunos momentos clave para iniciar.

\section{Definir la tipología del artículo}

Un aspecto para tener en cuenta a la hora de definir la tipología de su artículo tiene que ver con la organización y la proyección de su trabajo académico o investigativo. Es muy común que de un proyecto y de acuerdo con sus fases de implementación surja la necesidad de publicar un artículo de las tipologías artículo de resultado de investigación, artículo de revisión y artículo de reflexión. Así, por ejemplo, si su trabajo académico está en la fase de revisión de literatura y estructuración de antecedentes bibliográficos, es coherente que sistematice su proceso de revisión de literatura y pueda divulgar sus hallazgos mediante un artículo de revisión. De igual forma, si ya cuenta con resultados de su estudio puede apelar a la divulgación de ellos mediante un artículo de resultados de investigación. Por su parte, los artículos de reflexión presentan resultados de investigación terminada desde una perspectiva analítica, interpretativa o crítica.

El proceso de definición de la tipología del artículo también responde a criterios relacionados con la divulgación, la visibilidad y el impacto. La divulgación hace referencia a la firme intención de compartir con una comunidad puntual su trabajo 
académico; por tanto, es un momento de sumo cuidado que se articula al proceso de selección de la revista donde se espera que su documento sea publicado. De acuerdo con esa selección, existe mayor probabilidad de publicar un trabajo en una revista que tiene un público lector interesado en conocer la temática que usted abordó. Esto es fácil de identificar dado que todas las revistas declaran las temáticas a publicar, bien sea de manera permanente o por procesos de convocatoria. Por su parte, la visibilidad y el impacto están relacionados directamente con aspectos de indexación de la revista, entre ellos, la categoría e índice h de ella. Se presume que a mayor categoría e índice h existe mayor demanda de sometimiento de artículos en una revista, por lo tanto, los filtros de selección y aprobación del comité editorial para su posterior proceso de evaluación serán más rigurosos, atendiendo a consideraciones de calidad y selección/exclusión de los trabajos.

\section{Reconocer el contexto de publicación}

Se recomienda hacer una lectura previa de los trabajos y las tipologías de artículos que ha publicado la revista en sus correspondientes números. La revisión de los trabajos es un paso muy importante dado que ilustra y evidencia los estilos y los tipos, la calidad y el impacto de los trabajos que regularmente son publicados en la revista seleccionada.

\section{Identificar y seleccionar la revista}

Consiste en identificar la revista a la cual será sometido el artículo. Se hace necesario revisar el scope o alcance/enfoque de la revista para determinar si efectivamente el artículo elaborado cumple con las condiciones y las temáticas específicas de interés de la revista. Evite remitir un artículo a una revista que no guarde relación con el scope o alcance/enfoque.

La decisión de someter un artículo a una revista en una categoría de alto rango (tipo top A1, A2, en el ámbito colombiano, y cuartiles Q1, Q2 en el contexto internacional) también está sujeta a su experiencia y bagaje en el campo de las publicaciones científicas, por lo que se recomienda, en caso de ser la primera vez que postula un trabajo a una revista indexada, iniciar con aquellas que se encuentran en una categoría de menor rango.

\section{Preparar el artículo}

Esta etapa contempla tanto la revisión del documento de guía para autores/directrices para autores, como la adaptación del artículo a dicho documento. La guía para autores/directrices para autores debe seguirse al pie de la letra para garantizar que su trabajo cumpla con los requerimientos iniciales y pueda ser considerado por el comité editorial de la revista. Este es un documento de lectura obligatoria para la publicación.

\section{Enviar}

Prepare su trabajo teniendo en cuenta los momentos anteriormente descritos e inicie con el proceso de remisión según las indicaciones entregadas en la guía de autores. Siempre es importante tener un trato amable y respetuoso con el editor de la revista, a quien se recomienda enviar un mensaje poniendo en consideración su manuscrito.

Se aclara que las etapas aquí señaladas configuran un proceso cíclico para la organización de su trabajo, y, como toda actividad académica, puede continuar surtiendo un proceso iterativo.

\section{¿Qué aspectos debe considerar para escribir su trabajo?}

A continuación se enumera un listado de recomendaciones relacionadas con el proceso de construcción de los artículos:

1. Se recomienda que el título de su trabajo sea lo más corto posible (que no supere las 15 palabras, aunque esto puede variar de acuerdo con cada revista). En su título considere aspectos clave como las variables objeto de estudio y la población, además de lo llamativo y expectante para un público focalizado. 
2. Presente un resumen conciso y contundente. Para su elaboración, considere la incorporación de síntesis de apartados tales como objetivo, metodología, resultados y conclusiones, los cuales deben estar en un solo párrafo. No incluya citas o referencias de ninguna clase. La extensión de los resúmenes varía dependiendo de cada revista; no obstante, se recomienda una extensión no superior a las 250 palabras.

3. Tenga en cuenta identificar previamente las palabras clave de su trabajo en tesauros reconocidos. Se recomienda usar entre 3 y 5 palabras clave.

4. Es muy importante suministrar la información completa de los autores al interior del documento (en caso de así requerirse). No olvide indicar la filiación institucional y su código ORCID.

5. Escriba de manera clara y precisa presentando de entrada sus ideas centrales en cada párrafo. La información contenida en todo el documento debe mostrar organización, lógica interna y coherencia.

6. Procure emplear un lenguaje académico compartido por la comunidad científica a la cual se vincula su trabajo. Cuando use términos técnicos, verifique su correcta escritura.

7. Sea cuidadoso con el uso de la norma de citación solicitada por la revista. Es importante y necesario que su trabajo cuente con todas las citas y referencias de la propiedad intelectual empleada. Realice un adecuado parafraseo de las citas que revisa, evitando, en lo posible, el uso excesivo de citas literales a lo largo del artículo.

8. En cuanto al uso de tablas y figuras tenga en cuenta que son un recurso que ilustra con más detalle al lector; por tanto, es necesario garantizar su pertinencia al interior del documento. Todas las tablas y gráficas deben estar numeradas, tituladas y nombradas en el cuerpo del trabajo.

9. Para la introducción de su manuscrito incorpore lo siguiente: la pregunta problema, el objetivo/hipótesis del estudio y los antecedentes de orden internacional y nacional. Argumente sobre la importancia, la necesidad y la relevancia social de su trabajo para la comunidad internacional y nacional en la temática abordada. Evidencie en este apartado la relación existente entre su estudio, la teoría y las investigaciones previas. Además, se hace necesario que referencie autores de prestigio y reconocimiento que han escrito previamente sobre el tema central de su estudio. Incluya aspectos relacionados con la contribución y el aporte de su trabajo.

10. Presente su metodología de manera clara y precisa. No olvide tener en cuenta aspectos relacionados con el enfoque, el diseño, la población y la muestra, los instrumentos y el proceso de análisis e interpretación de datos. La metodología debe ser coherente y consistente con el objetivo de su trabajo, haciendo muy evidente el tipo de relación entre las variables abordadas. Recuerde tener presentes los aspectos éticos considerados para el tratamiento de los datos.

11. El apartado de resultados debe ser muy consistente, es decir, debe articularse necesariamente con los objetivos del estudio.

12. El apartado de discusión constituye un serio análisis que contrasta, entre otras fuentes, elaboraciones teóricas previas y resultados de otros estudios. Es importante que presente las limitaciones de su trabajo, así como también implicaciones teóricas y prácticas derivadas.

13. En las conclusiones incluya aspectos relacionados con aportes más importantes del trabajo, perspectivas, oportunidades para futuros estudios y dificultades.

14. Es muy importante que incluya en su trabajo aspectos relacionados con avales institucionales y reconocimiento a las entidades financiadoras en caso de tenerlos.

15. Los agradecimientos son opcionales.

16. Se recomienda emplear una buena bibliografía, preferiblemente proveniente de bases de datos de prestigio (WoS, Scopus, entre otras), y que esté actualizada.

17. Una vez terminado su trabajo siempre es muy importante compartirlo con algún miembro de la comunidad académica conocedor de la temática y, preferiblemente, que esta persona cuente con publicaciones en revistas indexadas para que pueda enriquecerlo con algunos comentarios y aportes. Una lectura externa, que realice un buen ejercicio crítico y reflexivo de su trabajo, siempre es necesaria para mejorar la calidad del documento.

18. Siempre es bueno que su trabajo pase por un proceso de corrección de estilo. Este aspecto, por lo general, posibilita una lectura clara, fluida y eficiente.

19. La participación y aporte de todos los autores en la construcción del trabajo debe ser compartida y aceptada por todos. 
20. Los tiempos de los procesos de gestión editorial en las revistas científicas suelen ser diversos; en algunos casos un poco más lento. Esto debido a la complejidad de las etapas involucradas en la cadena de gestión editorial. Por lo anterior, se recomienda ser paciente.

21. Finalmente, se aclara que un artículo sometido a una revista puede ser aceptado o rechazado; es decir, no existe compromiso de publicación del trabajo por parte de la revista. Por lo tanto, se sugiere ser muy riguroso con las orientaciones establecidas en la guía para autores y las que hemos entregado en este texto.

Si luego de seguir al pie de la letra cada uno de estos pasos su artículo es rechazado, no desestime su esfuerzo y compromiso; seguro será recompensado gratamente con el crecimiento y la experiencia del proceso vivido. Los informes de evaluación de los jurados y el editor son muy importantes para una nueva oportunidad durante el largo camino de la publicación científica. 